\title{
Ausbildungsvorbereitung von Jugendlichen mit besonderem Förderbedarf: das NRW-Modell
}

\begin{abstract}
Auch die sich seit einiger Zeit abzeichnende Entspannung auf dem Ausbildungsstellenmarkt wird nicht allen Jugendlichen einen nahtlosen Übergang von der Schule in Ausbildung ermöglichen. Absehbar bleiben insbesondere Jugendliche mit besonderem Förderbedarf weiterhin auf eine Zwischenstation der Ausbildungs- und Arbeitsmarktvorbereitung angewiesen, um ihre Chancen für eine Ausbildung nachhaltig zu verbessern. Dazu bedarf es jedoch einer institutionellen Neugestaltung, welche eine Antwort auf die zentralen organisatorischen wie auch methodisch-didaktischen Probleme in derartigen Bildungsgängen gibt. Worin diese Probleme bestehen und wie Lösungsansätze aussehen können, wird am Beispiel Nordrhein-Westfalen (NRW) gezeigt. ${ }^{\bullet}$
\end{abstract}

MARTIN BAETHGE, VOLKER BAETHGE-KINSKY

\section{Der Übergang von Schule in Ausbildung}

Seit über einem Jahrzehnt erweist sich der Übergang aus der allgemeinbildenden Schule in eine voll qualifizierende Berufsausbildung als die wohl problematischste Passage im deutschen Bildungssystem. Über Jahre hinweg mündeten etwa eine halbe Million Jugendliche bzw. 40 \% derjenigen, die einen Einstieg in eine Ausbildung oder Qualifizierung suchten, zunächst in eine der vielfältigen Übergangsmaßnahmen ein, die Voraussetzungen und Chancen der Jugendlichen für eine Ausbildung verbessern sollen, ihnen aber de facto keine klare Berufsbildungsperspektive eröffnen ( $\mathrm{Au}$ torengruppe Bildungsberichterstattung 2008, 2012). Auch wenn sich die Situation auf dem Ausbildungsstellenmarkt in den letzten Jahren - mehr aufgrund eines demografisch bedingten Nachfragerückgangs als wegen eines steigenden Ausbildungsplatzangebots - etwas entspannt hat, münden immer noch annähernd $30 \%$ der Neuzugänge zur beruflichen Bildung zunächst in Übergangsmaßnahmen ein - sowohl bundesweit (vgl. Autorengruppe Bildungsberichterstattung 2012, S. 102) als auch in NRW (vgl. IT NRW [o.J.] nach Baethge/Baethge-Kinsky 2012, S. 6).

Die Population des Übergangsbereichs ist nach sozialen Merkmalen nicht genau aufzuschlüsseln, sodass auch der hier besonders interessierende Anteil der Jugendlichen mit besonderem Förderbedarf nicht exakt bezifferbar ist, ganz abgesehen davon, dass auch die Kategorie „besonderer Förderbedarf" nicht eindeutig bestimmt ist. Bis heute gibt es keine allgemein verbindliche und eindeutige gesetzliche Definition der „Jugendlichen mit besonderem Förderbedarf“. Folgt man der in der wissenschaftlichen Begleitfor-

- Im Zentrum dieses Beitrags stehen die Ergebnisse einer qualitativen explorativen Studie, die die Autoren im Auftrag des nordrhein-westfälischen Ministeriums für Schule und Weiterbildung an Berufskollegs in NRW durchgeführt haben. Ziel der Untersuchung war es, die Voraussetzungen und Probleme eines neuen Typs von Ausbildungsvorbereitung, der ab 2013 eingeführt wird, zu ermitteln. Die explorative Studie wurde vom Soziologischen Forschungsinstitut (SOFI) an der Universität Göttingen an zwölf systematisch ausgewählten Berufskollegs in NRW durchgeführt und zwar in Form von Expertengesprächen mit Schulleitungen, Fokusgruppen-Diskussionen mit Lehrkräften sowie Sozialarbeitern und Sozialarbeiterinnen, die Erfahrungen in BOJ-Klassen (Berufsorientierungsjahr) bzw. in Klassen für Schüler ohne Berufsausbildungsverhältnis (KSoB) hatten. Ferner wurden Dokumentenanalysen vorgenommen (vgl. im Einzelnen Baethge/Baethge-Kinsky 2012, S. 22ff.). Wünschenswert wäre es gewesen, wenn man auch die Jugendlichen selbst, Betriebe und Träger hätte einbeziehen können. Das aber hätte den zeitlichen und personellen Rahmen dieser Analyse gesprengt, zumal intendiert war, dass die Ergebnisse bis zum Start der Neuorganisation der Ausbildungsvorbereitung vorliegen sollten. Der Leser kann selbst beurteilen, ob diese Einschränkung die Ergebnisse wesentlich beeinträchtigt hat. 
schung zur Benachteiligtenförderung entwickelten Definition für „Benachteiligte“ (vgl. BMBF 2005; Plicht 2010), so würden Jugendliche mit besonderem Förderbedarf in den Umkreis einer der drei folgenden Arten der Benachteiligung beim Übergang in Ausbildung und Beschäftigung gehören:

- Soziale Benachteiligung vor allem aufgrund sozialer Herkunft, schulischer Vorbildung, Geschlecht und Migrationshintergrund;

- Lernbeeinträchtigungen, die sowohl die kognitiven Lernvoraussetzungen als auch Verhaltensauffälligkeiten betreffen sowie

- Marktbenachteiligungen, die sich aus der Struktur des Berufsbildungs- und Beschäftigungssystems ergeben und auf Verdrängungsprozesse beim Wettbewerb um knappe Ausbildungsplätze verweisen (BMBF 2005, S. 12ff.).

Die vorstehende Definition der im Übergang zur Berufsausbildung Benachteiligten ist allerdings insbesondere mit dem Einbezug der „Marktbenachteiligten“ zu weit gefasst, um „Jugendliche mit besonderem Förderbedarf“ hierunter subsumieren zu können. Letztere sind nicht von Marktungleichgewichten her zu definieren, selbst wenn diese in der Realität auch sie beeinträchtigen. Vielmehr sind es vor allem persönliche soziale und biografische Merkmale, die als Barriere für die Aufnahme einer Ausbildung wirken. Sicherlich gehört zu dieser Gruppe die Mehrheit der Jugendlichen ohne Schulabschluss; vermutlich wird man aber auch einen Teil von Jugendlichen mit dazurechnen müssen, die nach den internationalen Leistungsstudien am Ende der Sekundarstufe I die für die Aufnahme einer Berufsausbildung erforderlichen Kompetenzen nicht erreichen; ${ }^{2}$ schließlich wird man Jugendliche einbeziehen müssen, die in ihrer psycho-sozialen Entwicklung die für eine Ausbildung erforderlichen Verhaltensdispositionen noch nicht ausgebildet haben. ${ }^{\circ}$

Von der derzeitigen Entspannung auf dem Ausbildungsmarkt haben offensichtlich auch bildungsbenachteiligte Jugendliche profitiert. Ein Indiz dafür ist der überproportionale Rückgang der Einmündungsquote von Jugendlichen ohne Hauptschulabschluss ins Übergangssystem, der in NRW zwischen 2006 und 2010 zwölf Prozentpunkte ausmacht (Abbildung 1). Es erscheint wahrscheinlich, dass im andauernden demografisch bedingten Abschwung der Ausbildungsnachfrage sich die Zahl der "Marktbenachteiligten“ weiter verringern und sich die Schülerschaft des Übergangsbereichs zunehmend auf Jugendliche mit besonderem Förderbedarf konzentrieren wird. Auch dies wird mit begrenzten regionalen Differenzen nicht allein in NRW, sondern bundesweit der Fall sein, wobei die regionalen Differenzen die Eingliederungschancen für die Jugendlichen in den auch heute wirksamen Bandbreiten modifizieren (vgl. Blien/Hirschenauer 2005).
ABB. 1

\section{Neuzugänge in berufliche Ausbildung nach schulischer Vorbildung in NRW 2006, 2008 und 2010}

Angaben in Prozent $\square$ Duales System $\square$ Schulberufssystem Übergangssystem

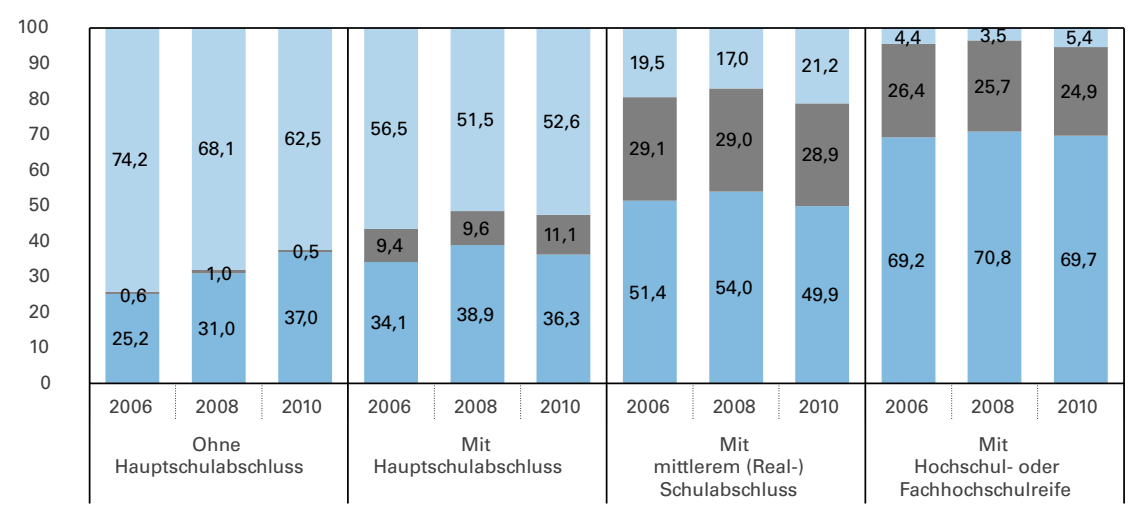

Quelle: Statistisches Bundesamt (2011, 2012); IT NRW (o.J.); Berechnungen der Autoren.

\section{Mangelnde Effizienz und institutionelle Heterogenität des Übergangssystems}

Mehr oder weniger alle Maßnahmetypen des Übergangssystems sind darauf ausgerichtet, die persönlichen Voraussetzungen von Schulabgängern für die Aufnahme einer voll qualifizierenden Ausbildung zu verbessern und ihnen den Übergang zu ermöglichen; hierzu sollen eine Erhöhung ihrer kognitiven Kompetenz, berufsorientierende praktische Erfahrungen in Betrieben oder Lehrwerkstätten sowie eine Stärkung ihrer Ausbildungsmotivation dienen.

(2) Die Organisation für wirtschaftliche Zusammenarbeit und Entwicklung (OECD) definiert die Kompetenzstufe 2 als „Basisniveau der Lesekompetenz, ab dem die Schülerinnen und Schüler jene Lesekompetenzen unter Beweis zu stellen beginnen, die sie befähigen werden, effektiv und produktiv am Leben teilzuhaben" (OECD 2010, S. 172). Auch wenn sich diese Expertise die OECD-Einschätzung für die Berufsbildung nicht zu eigen macht, wird man zugestehen müssen, dass es kognitive Mindeststandards für die Absolvierung einer Berufsausbildung gibt.

(3) Aus untersuchungstechnischen Gründen ist in der empirischen Studie eine pragmatische Definition gewählt, die sich auf die Schülerinnen und Schüler der heutigen Klassen im „Berufsorientierungsjahr" (BOJ) und in den „Klassen für Schüler ohne Berufsausbildungsverhältnis" (KSoB) in NRW bezieht. Es kann erwartet werden, dass in ihnen der Großteil der Jugendlichen, denen in der Literatur besonderer Förderbedarf zugesprochen wird, versammelt ist. 
TABELLE 1

Berufsschulanfänger ohne Hauptschulabschluss und Absolventen mit erreichtem Hauptschulabschluss des Schuljahres 2009/2010 in unterschiedlichen Typen der Ausbildungsvorbereitung in NRW

Angaben in absoluten Zahlen und Prozent

\begin{tabular}{|c|c|c|c|}
\hline Bildungsgang/Maßnahme & $\begin{array}{l}\text { Berufsschulanfänger ohne } \\
\text { Hauptschulabschluss }^{1}\end{array}$ & $\begin{array}{l}\text { Absolventen mit erreichtem } \\
\text { Hauptschulabschluss }\end{array}$ & $\begin{array}{l}\text { Erfolgsquote } \\
\quad-\text { in } \%-\end{array}$ \\
\hline Berufsorientierungsjahr (BOJ) & 3.632 & 1.540 & 42,4 \\
\hline $\begin{array}{l}\text { Klassen für Schüler ohne Berufsausbil- } \\
\text { dungsverhältnis (KSoB)-TZ, darunter... }\end{array}$ & 10.941 & 1.202 & 11,0 \\
\hline ...Berufsvorbereitungsjahr (BVB) & 5.320 & 744 & 14,0 \\
\hline ...Werkstattjahr & 1.451 & 260 & 17,9 \\
\hline ...Jungarbeiter/Ausbildungslose & 4.170 & 198 & 4,7 \\
\hline KSoB-VZ & 2.186 & 443 & 20,3 \\
\hline Ausbildungsvorbereitung insgesamt & 16.759 & 3.185 & 19,0 \\
\hline
\end{tabular}

Schüler im 1. Schuljahr zum Stichtag 15.10.2009.

${ }_{2}^{2}$ Anteil der Absolventen mit erreichtem Hauptschulabschluss an den Berufsschulanfängern ohne Hauptschulabschluss.

ABB. 2

\section{Verteilung der Neuzugänge auf die unterschiedlichen Formen der Ausbildungsvorbereitung in NRW 2006, 2008 und 2010}

Angaben in absoluten Zahlen

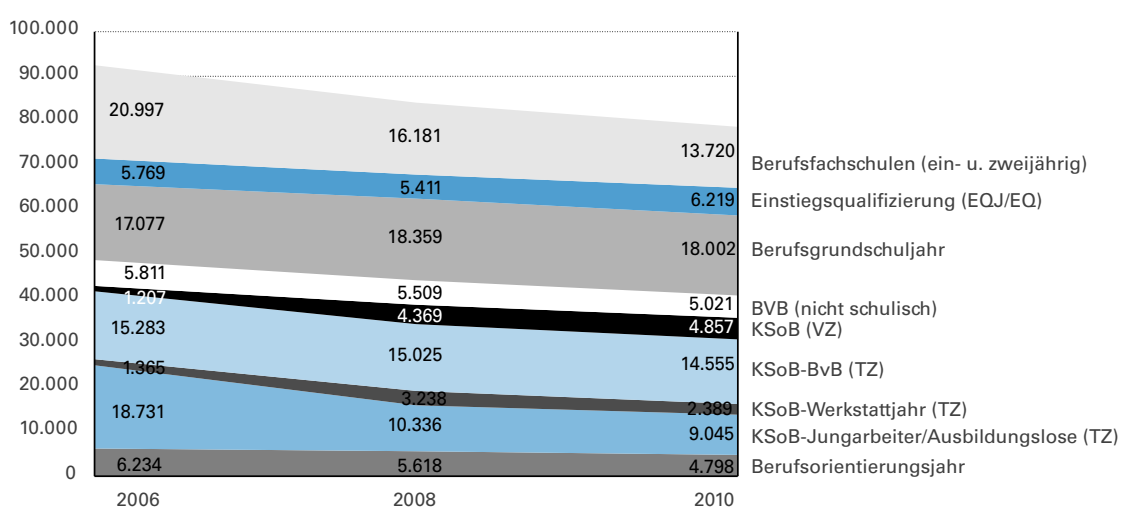

Quelle: Statistisches Bundesamt (2011, 2012); IT NRW (o.J.); Berechnungen der Autoren. richterstattung 2008, S. 164ff.; Beicht et al. 2008). Hohe Abbrecherquoten bei berufsvorbereitenden (BVB)-Maßnahmen der Bundesagentur für Arbeit (bundesweit $31 \%$, vgl. Plicht 2010) und beim „Werkstattjahr“, das aus Mitteln des Europäischen Strukturfonds (ESF) und Landesmitteln finanziert wird (Abbruchsquote hier beispielsweise in NRW 2007 - 2009 über 50 \%, davon zwei Drittel ohne Anschlussperspektive, vgl. Eckhardt/Sommer 2011), bestätigen das kritische Urteil. Betrachtet man nur die Verbesserung der Allgemeinbildung in dem Sinne, dass ein zunächst verfehlter Hauptschulabschluss nachgeholt wird, so fällt die Bilanz der Ausbildungsvorbereitung nicht besser aus: Nach den Daten für NRW haben $19 \%$ der Jugendlichen, die im Schuljahr 2009/2010 ohne Hauptschulabschluss in die Ausbildungsvorbereitung eingetreten sind, diese mit einem frisch erworbenen Hauptschulabschluss verlassen. Hierbei schneidet das Berufsorientierungsjahr (BOJ) mit einer Erfolgsquote von über $42 \%$ noch vergleichsweise gut ab; die anderen Typen der Ausbildungsvorbereitung und insbesondere die Teilzeit (TZ)-Klassen für Jugendliche mit („Jungarbeiter") und ohne Arbeits-bzw. Ausbildungsverhältnis weisen deutlich niedrigere Erfolgsquoten auf ( $\mathrm{Ta}$ belle 1).

Jenseits der sozialstrukturellen und biografischen Merkmale der Jugendlichen, die in den Übergangsverläufen sichtbar werden (vgl. Beicht et al. 2008, S. 260ff.), existiert über die institutionellen Ursachen für die begrenzte Effektivität des Übergangssystems wenig Transparenz (vgl. beispielhaft Hälfte und zwei Dritteln der Jugendlichen im Übergangssystem gelingt in den ersten 30 Monaten nach Schulabschluss der Übergang in eine voll qualifizierende Ausbildung. Die Probleme kumulieren bei den Jugendlichen mit und ohne Hauptschulabschluss, und noch einmal verstärkt bei Migrantenkindern (vgl. Autorengruppen Bildungsbe-
(4) Beicht et al. (2008, S. 305f.) verweisen zu Recht darauf, dass individuelle Effekte wie Weiterentwicklung und Stärkung persönlicher Identität durch die qualitativen Indikatoren nicht erfasst würden. 
ZEW et al. 2010). Evaluationsstudien beziehen sich jeweils nur auf einzelne Maßnahmetypen und sind selten repräsentativ. Transparenz herzustellen, wird nicht zuletzt durch die institutionelle Heterogenität erschwert, die das Übergangssystem auszeichnet. Sofern sich ein System dadurch auszeichnet, dass seine einzelnen Elemente systematisch aufeinander bezogen und durch Ausrichtung auf ein gemeinsames Ziel hin koordiniert werden, ist das Übergangssystem letztlich gar kein System, sondern ein Konglomerat von nach jeweils eigenen Regulationsprinzipien funktionierenden Maßnahmentypen, deren Gemeinsamkeit in der Klientel und jeweils unterschiedlichen Übergangszielsetzungen besteht.

Die institutionelle Heterogenität wird an den unterschiedlichen Formen der Ausbildungsvorbereitung in NRW deutlich (Abbildung 2). Die Ausbildungsvorbereitung der Jugendlichen im Übergangssystem erfolgt nicht in einem nach einheitlichen Zielsetzungen und Durchführungsrichtlinien konzipierten und organisierten, homogenen Bildungsgang. Sie zerfällt vielmehr in unterschiedliche Formen der Ausbildungsvorbereitung mit je eigenen Organisationsprinzipien:

- Berufsorientierungsjahr (BOJ), ${ }^{\odot}$

- Voll- und Teilzeitklassen für Jungarbeiter und Jugendliche ohne Ausbildungsvertrag (KSoB),

- Jugendliche in trägergestützten Landesprogrammen („Werkstattjahr“),

- Bundesprogramme der Bundesagentur für Arbeit (BA) wie Berufsvorbereitungsmaßnahmen (BvB),

- Berufsgrundschuljahr (BGJ),

- Einstiegsqualifizierung,

- Berufsfachschulen.

Diese Berufsvorbereitungstypen, die die bundesweite Struktur des Übergangssystems spiegeln,

- sind mit „Unschärfen“ in der Adressierung (Überlappung von Zielgruppen) versehen,

- liegen in unterschiedlichen Verantwortlichkeiten institutioneller Akteure (Berufsschulen, Bundesagentur für Arbeit, Maßnahmeträger, Betriebe), was die Zuweisungsprozesse wie auch die Durchführung der Bildungsgänge anbelangt, - variieren tendenziell in der Verbindlichkeit und Dichte fachlicher und pädagogischer Zielvorgaben (Ausnahme: Nachholen des Hauptschulabschlusses) und

- weisen jenseits der durch Landesrecht einheitlich normierten Unterrichtsumfänge, der Notenmaßstäbe für das Erteilen des Hauptschulabschlusses und der Personalbemessung zum Teil erhebliche Unterschiede in den Standards der Durchführung auf (Verwendung von Qualifizierungsbausteinen bzw. -modulen; Verbindlichkeit der Ableistung betrieblicher Praktika, deren Umfang und zeitliche Lage) (vgl. MSW 2011a, 2011b; BA 2009; AVO-RL 2005).
Wie die Entwicklung der letzten Jahre zeigt, behalten alle diese Formen der Ausbildungsvorbereitung in NRW Gewicht - trotz eines zwischen 2006 und 2010 beobachtbaren Rückgangs der Teilnehmer um über $16 \%$ : Besonders stark fällt hier der Rückgang bei den Neuzugängen in BOJ (-23\%) und in die Teilzeit-KSoB-Klassen für Jungarbeiter/Ausbildungslose (-52 \%) aus. Demgegenüber bleiben die KSoBBvB in etwa stabil, die Teilnehmerzahl beim KSoB-Werkstattjahr nimmt zu; schließlich hat sich die Zahl der Neuzugänge in der Vollzeit-Variante der KSoB-Klassen im gleichen Zeitraum mehr als verdreifacht (Abbildung 2).

\section{Reduzierung institutioneller Hetero- genität in der Ausbildungsvorbe- reitung: der NRW-Ansatz}

Angesichts der institutionellen Heterogenität der Übergangsmaßnahmen und der begrenzten Erfolge der Ausbildungsvorbereitung erscheinen Ansätze zur Reduzierung der institutionellen Zersplitterung im Übergangsbereich als wichtiger Schritt zur Verbesserung der Ausbildungsvorbereitung. ${ }^{\odot}$ Einen solchen Schritt geht das Ministerium für Schule und Weiterbildung (MSW) in NRW mit dem Plan, in den Berufskollegs in NRW eine neue integrierte Form von Berufsvorbereitung ab 2013 einzuführen.

Der Plan des Ministeriums sieht vor, die unterschiedlichen Formen der Berufsvorbereitung in den Kollegs auf eine einheitliche Grundlage zu stellen und einen neuen Typ von einjähriger Ausbildungsvorbereitung zu implementieren, der sich an Jugendliche mit besonderem Förderbedarf richtet und in dem das bisherige Berufsorientierungsjahr (BOJ) und die Klassen für Schüler ohne Ausbildungsvertrag (KSoB) auf eine neue didaktische und organisatorische Grundlage gestellt werden sollen. Ziel ist, durch die Vermittlung von Kenntnissen und Fertigkeiten in Form von (zertifizierbaren) Qualifizierungsbausteinen aus einem Berufsfeld oder mehreren und durch die Erweiterung allgemeinbildender Kompetenzen die Vorbereitung auf eine Berufsausbildung so zu verbessern, dass der Abschluss

5 Das BOJ in NRW entspricht dem in fast allen anderen Bundesländern existierenden Berufsvorbereitungsjahr.

6 Wir beziehen uns nur auf das Übergangsmanagement jenseits der allgemein bildenden Schulen. Die in den letzten Jahren forcierten politischen Anstrengungen, in den Abschlussklassen der Sekundarstufe I neue Formen der Berufsorientierung zu praktizieren (wie im Jobstarter-Programm), werden hier ausgeklammert, auch wenn sie einen wichtigen Beitrag zur Bewältigung der Übergangsproblematik leisten (vgl. Lippegaus-Grünau et al. 2011; Kupka/ Wolters 2010; Krüger-Charlé et al. 2011). 
des Bildungsgangs einen direkten Anschluss an eine Berufsausbildung wie auch (gegebenenfalls) den Erwerb des Hauptschulabschlusses ermöglicht. Die Ausbildungsvorbereitung soll aber auch Perspektiven für einen Übergang in eine gestufte Berufsfachschule mit der Option des Erwerbs anerkennungsfähiger Ausbildungsbausteine und des mittleren Schulabschlusses eröffnen (vgl. MSW 2011a, Anlage A).

Als Aufnahmevoraussetzung gilt: Es sollen Schüler sein, die in der Regel keinen oder einen schwachen Hauptschulabschluss haben; eine einvernehmliche Anschlussvereinbarung soll bei der Wahl des Berufsbereichs Berücksichtigung finden.

Die Klassen sollen nach Berufsbereichen gebildet werden. Vorgesehen sind zwei Varianten dieses Grundtyps, die die unterschiedlichen Einbindungen von Jugendlichen in den Arbeitsmarkt bzw. in arbeitsmarktpolitische Maßnahmen berücksichtigen: Der Typ A ist für diejenigen Jugendlichen vorgesehen, die nur begrenzt für eine Vollzeitbeschulung zur Verfügung stehen, weil sie entweder einer sozialversicherungspflichtigen Beschäftigung nachgehen oder aber an drei Tagen in der Woche in Trägermaßnahmen betreut werden; dieser Typ sieht eine Teilzeitbeschulung durch das Berufskolleg an zwei Wochentagen vor und umfasst die „alten“ KSoB-Klassen für BvB, Werkstattjahr und „Jungarbeiter“. Der Typ B integriert jene „alten“ Bildungsgänge, in denen sich - in der Regel in Vollzeit (bei den "Ausbildungslosen“ aber auch nur in Teilzeit) - Bildung und schulfachliche Begleitung der Jugendlichen in der Verantwortung des Berufskollegs vollzieht (Vollzeit-KSoB-Klassen und BOJ). Dieser Typ sieht ein an drei Tagen in der Woche abzuleistendes Praktikum und ergänzend eine zweitägige Beschulung vor - durchgängig in der verantwortlichen Betreuung durch die Berufskollegs.

Im Vergleich mit anderen Initiativen wie „Übergänge mit System“ von zehn Bundesländern, der Bundesagentur für Arbeit und der Bertelsmann Stiftung (Autorengruppe BIBB/Bertelsmann Stiftung 2011), die auf der Strukturebene ansetzen, verbindet der Plan des MSW-NRW die (schulische) Strukturebene mit der Prozessebene der Durchführung, auf der sich die Qualität berufsvorbereitender Kompetenzen der Jugendlichen entscheidet und deren Neugestaltung den eigentlichen Fortschritt des NRW-Ansatzes darstellt.

\section{Zur Situation der Ausbildungsvorbe- reitung von Jugendlichen mit besonderem Förderbedarf in NRW}

Weil sich mit einer gesetzlichen Änderung nicht automatisch die Prozesse der Ausbildungsvorbereitung verändern, erschien dem MSW eine wissenschaftliche Klärung der Be- dingungen und Probleme der aktuellen Ausbildungsvorbereitung an den Berufskollegs notwendig, da sich in ihnen wichtige Handlungserfordernisse für die Implementation des neuen Berufsvorbereitungskonzepts finden lassen müssten. Die Ergebnisse der explorativen Studie (vgl. Fußnote 1) an Berufskollegs werden im Folgenden unter vier Aspekten skizziert: Heterogenität der Schülerschaft (4.1), organisatorische und didaktische Probleme der Ausbildungsvorbereitung (4.2), institutionelle Formen (4.3) und Professionalisierungsprobleme beim Lehrpersonal (4.4).

\subsection{Heterogenität der Schülerschaft als Problem}

Die aus anderen (mit Stichproben von Jugendlichen arbeitenden) Übergangsstudien bekannten Befunde zu den Merkmalen von Jugendlichen in der Ausbildungsvorbereitung (vgl. u.a. Beicht et al. 2008; Solga et al. 2010) treten auch in den Schilderungen der Lehrkräfte als zentraler Bezugspunkt ihrer pädagogischen Arbeit zutage: Die in den zwölf Berufskollegs geführten Expertengespräche mit Schulleitungen und Fokusgruppen-Diskussionen mit Lehrkräften und Sozialarbeitern und Sozialarbeiterinnen machen nicht nur deutlich, dass die oben herausgearbeiteten sozialen Profile und Verhaltensmerkmale in ähnlicher Weise auch bei den Jugendlichen der BOJ- und KSoB-Klassen der Berufskollegs zu finden sind, sondern verweisen auch auf das damit verbundene eigentliche Problem: Neben unterschiedlichen Vorbildungsniveaus gibt es eine hochgradige Durchmischung von sozialstrukturellen, (bildungs-)biografischen sowie psychosozialen und kognitiven Lernbeeinträchtigungen der Schülerinnen und (mehrheitlich) Schüler in allen Typen der Ausbildungsvorbereitung, die den Unterrichtsalltag prägen und eine andauernde Herausforderung darstellen.

Auch wenn die in einer Fokusgruppe vorgestellte Unterscheidung in drei Schülertypen von Jugendlichen mit besonderem Förderbedarf - „schlaue, aber sozial entgleiste; spät zugewanderte mit Sprachproblemen; lernbeeinträchtige, aber lernwillige“ - sicherlich nicht die Realität aller Kollegs und ihrer Bildungsgänge in der Ausbildungsvorbereitung trifft, spiegelt sie doch ziemlich gut die unterschiedlichen Lernvoraussetzungen und -motivationen, mit denen sich die Lehrerschaft konfrontiert sieht: Unterschiede in der allgemeinen Leistungsfähigkeit aufgrund der Bildungsferne des Elternhauses, eklatante Schwächen in Deutsch und Mathematik, stark ausgeprägte, gesonderte Förderung erfordernde kognitive Defizite stellen die eine Seite der Heterogenität in den BOJ- und KSoB-Klassen dar. Die andere Seite sind Verhaltensauffälligkeiten, die sich nicht nur in aggressivem Verhalten gegenüber Lehrkräften aufgrund negativer Erfahrungen in der allgemeinbildenden Schule ausdrücken, sondern auch darin, dass sich die Schüler aufgrund schwerer psychischer Traumata („Flüchtlinge“), Autismus, krimineller Karrieren oder biografisch angelegter Orientierungslosigkeit schwertun, sich in eine wie auch immer strukturierte Lernwelt einzufügen. 
Nach unseren Erhebungen spielen diese Heterogenitätsdimensionen in allen Kollegs und in allen Formen der Ausbildungsvorbereitung eine Rolle; Unterschiede liegen eher in der Stärke und der Häufigkeit des Auftretens bestimmter Merkmale (abweichendes Verhalten, Lerneinschränkungen). Nicht der Umstand, dass die Schülerschaft der Ausbildungsvorbereitung sehr heterogen ist, stellt das entscheidende Problem dar; relevant ist vielmehr, dass eine vieldimensionale (soziale, kognitive, motivationale) Heterogenität im Mikrokosmos der Klasse kumulativ auftritt und täglich von einzelnen Lehrkräften bewältigt werden muss.

\subsection{Organisatorische und methodisch- didaktische Probleme der Ausbildungs- vorbereitung}

Die in den Expertengesprächen und Fokusgruppen-Diskussionen geschilderten organisatorischen und methodischdidaktischen Probleme der derzeitigen Ausbildungsvorbereitung in NRW lassen sich in fünf zentralen Faktoren, die eine erfolgreiche Durchführung der Ausbildungsvorbereitung beeinträchtigen, bündeln:

(1) Der erste Faktor betrifft die Zeitorganisation. Angesichts des realen Förderbedarfs vieler Jugendlicher erscheint ein Jahr zu kurz. Hinzu kommt ein zur Erreichung der vorgegebenen Ziele schwer auflösbares Spannungsverhältnis zwischen der gleichzeitigen Realisierung unterschiedlicher Ziele (Verbesserung kognitiver Fähigkeiten und Vermittlung berufspraktischer Erfahrungen) und den durch die gesetzlichen Vorgaben (Stundentafel!) eingeschränkten Möglichkeiten einer flexiblen, an den Interessenlagen, Kompetenzen und Schwächen der Jugendlichen ansetzenden Unterrichtsorganisation (Gestaltung der Inhalte sowie Anordnung der Lernorte).

(2) Interne organisatorische Probleme, die Ausbildungsvorbereitung als berufs(bereichs)bezogenen Unterricht anzulegen, stellen sich zum einen in zu hohen Klassenstärken, die dem Bedarf dieser Klientel an persönlicher Beziehung und individueller Betreuung (auch außerhalb des Unterrichts) nicht entspricht; zum anderen in der Sicherstellung motivierten und qualifizierten d. h. kompetenten Personals; schließlich auch in der Bereitstellung der benötigten technischen und räumlichen Kapazitäten (Unterrichtsräume und Werkstätten).

(3) Da Ausbildungsvorbereitung zumeist nicht nur in Kollegs stattfindet, spielen Probleme externer Kooperation eine wichtige Rolle: Hierzu zählt zum einen der Mangel an Informationen über Interessen, Leistungsvoraussetzungen und -einschränkungen der potenziellen Schülerschaft aufgrund unzureichend ausgebauter Kontakte zu abgebenden Schulen, Schülern und Elternschaft. Zum anderen zählen hierzu auch die teilweise mühsamen Kooperationen mit den jeweiligen Trägern im Rahmen von Werkstattjahr und
BvB-Maßnahmen, die insbesondere eine sinnvolle Abstimmung von Lernsequenzen und Lerninhalten zwischen dem Personal von Kollegs und Trägern erschweren - zumal, wenn unterschiedliche pädagogische Konzepte verfolgt werden.

(4) Als sehr hoch wird oft der Aufwand an Zeit und Nerven geschildert, einen halbwegs ungestörten und konfliktarmen, leistungsdifferenzierten Unterricht im Rahmen eines auf Kontinuität angelegten Klassenverbundes sicherzustellen; dieser Aufwand wird zudem insbesondere durch die hohe Fluktuation bei den Schülern aus den trägerstützten Maßnahmen (BvB, Werkstattjahr) wie auch durch Absentismus immer wieder konterkariert.

(5) Schon zu Beginn des Schuljahrs, aber auch in dessen Verlauf (z. B. bei absehbarer Gefährdung des angestrebten Hauptschulabschlusses), treten immer wieder Motivationsund Beteiligungsprobleme, insbesondere im theoretischen Berufsschulunterricht auf.

\subsection{Unvollständige Institutionalisierung der Ausbildungsvorbereitung}

Die aufgeführten Probleme werden heute in den Berufskollegs - so gut es geht - vor allem durch ein hohes pädagogisches Engagement der Lehrerinnen und Lehrer gelöst, ohne dass die betroffenen Lehrkräfte mit den Lösungen durchweg zufrieden wären. Sie verweisen in den Gesprächen und Diskussionen immer wieder auf institutionelle Begrenzungen ihrer Arbeit. Die in den Äußerungen artikulierten Arbeitsformen, die von Kolleg zu Kolleg differieren, lassen sich idealtypisch zwischen zwei Polen der Institutionalisierung $^{(0}$ - relativ gering und relativ stark ausgeprägte Institutionalisierung - verorten, an deren Vergleich die Schwächen der Institutionalisierung der Berufsvorbereitung insgesamt sichtbar werden.

\subsubsection{Der Typ einer relativ gering ausgeprägten Institutionalisierung}

Dieser Typ zeichnet sich dadurch aus, dass die jeweiligen Antrittsgesetze für einen bestimmten Typus von Ausbildungsvorbereitung (Heterogenität der jeweiligen Schülerschaft, institutionelle Zieldefinition und Vorgaben für die Durchführung) und die daraus resultierenden Probleme mehr oder weniger im Rahmen der traditionellen Kollegorganisation „mitlaufen“ - ohne größere Folgen für die kolleginterne Organisation und die methodisch-didaktische Konzeption des einzelnen Bildungsgangs bzw. Bildungsgangtyps.

(7) Idealtypisch heißt hier, dass die beiden in Übersicht $1 \mathrm{ab}$ gebildeten Pole aus den Gesprächen abstrahierte Konstrukte sind, denen sich die Organisationsrealität der Kollegs jeweils nur immer mehr oder weniger annähert. 
Formen (Typen) der Institutionalisierung der Ausbildungsvorbereitung in Berufskollegs

Typen nach Grad der Institutionalisierung

\begin{tabular}{|c|c|c|}
\hline $\begin{array}{l}\text { Dimensionen der } \\
\text { Institutionalisierung }\end{array}$ & $\begin{array}{l}\text { Relativ gering ausgeprägte } \\
\text { Institutionalisierung }\end{array}$ & $\begin{array}{l}\text { Relativ stark ausgeprägte } \\
\text { Institutionalisierung }\end{array}$ \\
\hline Einbau in Kollegorganisation & en passant & (ansatzweise) eigene Abteilung \\
\hline \multirow{2}{*}{ Einsatz der Lehrkräfte } & $\begin{array}{l}\text { an mehreren Lernorten (z. B. Fachklassen und } \\
\text { Ausbildungsvorbereitung) }\end{array}$ & (tendenziell) ein Lernort (Ausbildungsvorbereitung) \\
\hline & fluktuierend mit zumeist begrenzter Stundenzahl & kontinuierlich (in der Regel) Vollzeit-Ausbildungsvorbereitung \\
\hline Lernziel-Definition & $\begin{array}{l}\text { (formale) Übernahme der gesetzlich vorgegebenen } \\
\text { Lernziele }\end{array}$ & $\begin{array}{l}\text { Individualisierung der vorgegebenen Lernziele nach } \\
\text { Bedingungen der Schüler }\end{array}$ \\
\hline $\begin{array}{l}\text { Interne Differenzierung des } \\
\text { Unterrichts }\end{array}$ & relativ gering (überwiegend Klassenverband) & Bildung kleiner Gruppen und individuelle Arbeit \\
\hline \multirow{2}{*}{ Spektrum der Lerngegenstände } & $\begin{array}{l}\text { begrenzt, wenig zusätzliche inhaltliche } \\
\text { Angebote }\end{array}$ & $\begin{array}{l}\text { erweitertes, auf individuelle Bedürfnisse zugeschnittenes } \\
\text { Angebot von Lerngegenständen }\end{array}$ \\
\hline & $\begin{array}{l}\text { Ausdehnung von Praktika und } \\
\text { Werkstattunterricht }\end{array}$ & $\begin{array}{l}\text { Ausdehnung von Praktika und anderen Praxisformen in und } \\
\text { außerhalb des Kollegs }\end{array}$ \\
\hline Betreuung der Schüler & im Rahmen des Unterrichts in Klassen & $\begin{array}{l}\text { Begleitung der unterrichtlichen und - soweit möglich - } \\
\text { außerunterrichtlichen (lebensweltlichen) Abläufe }\end{array}$ \\
\hline
\end{tabular}

Bezogen auf die kolleginterne Organisation existiert weder eine abteilungsmäßige Separierung der Ausbildungsvorbereitung (als Vorbedingungen für eine Professionalisierung), noch ist ein aktiver Umgang mit dem Heterogenitätsproblem zu beobachten: Der Zustrom von Jugendlichen in die jeweiligen Typen und Klassen der Ausbildungsvorbereitung wird kaum gefiltert oder gar direkt gesteuert, sondern mehr oder minder stillschweigend als kaum beeinflussbar hingenommen. Die Unterrichtsversorgung in den BOJ- und KSoB-Klassen wird in periodischem Wechsel von Lehrkräften wahrgenommen, die ansonsten in Fachklassen eingesetzt sind. Eine qualifikatorische Spezialisierung auf die besondere Klientel bleibt dem individuellen Engagement und mehr oder weniger naturwüchsigen Austausch unter Kollegen vorbehalten.

Die Probleme, die aus der gesetzlichen Zieldefinition herrühren, werden in der Regel dort angegangen, wo dies über eine im Rahmen der Stundentafel zulässige Ausdifferenzierung bestehender Bildungsangebote möglich ist, beispielsweise durch Schwerpunktbildungen in bestimmten Bereichen der Allgemeinbildung (Mathematik, Deutsch). An den Zielen selbst wird - allerdings in einem eher formalen Sinn, der das Scheitern eines Teils der Schüler in Kauf nimmt - festgehalten.

Obwohl die Probleme einer einheitlichen Unterrichtsdurchführung wahrgenommen werden, bleibt der Unterricht im Klassenverband unangetastet. Die durchaus als wünschenswert angesehene Stärkung von Praxisanteilen beschränkt sich auf eine, über das vorgegebene Mindestmaß eher vorsichtig hinausgehende Ausdehnung der Zeiten in
Werkstatt oder im betrieblichen Praktikum. Eine weiterreichende Verzahnung von Theorie und Praxis (insbesondere in der Verbindung von allgemeinbildenden Inhalten mit Aufgabenstellungen aus der Werkstatt) wird hier unter Verweis auf die Grenzen der Lernfelddidaktik eher skeptisch eingeschätzt. Zwar werden auch in diesem Typ die Lernthemen durch Zusatzangebote ausgeweitet, diese erschöpfen sich aber häufig in wenigen Angeboten, die eher eine Lösung von disziplinarischen Unterrichtsproblemen (z. B. Trainingsräume zur Bearbeitung von Konflikten) darstellen, als dass sie Antworten auf die lebensweltlichen und -praktischen Fragen von Jugendlichen geben.

Betrachtet man bei diesem Typ die Organisation der Unterrichtsdurchführung in all ihren Facetten, dann orientiert diese sich mehr an den Usancen eines Unterrichts, den man so auch in den Fachklassen des dualen Systems wiederfinden könnte: Die Lernzieldefinition bleibt auf den vorgegebenen Richtlinien-Rahmen beschränkt, wird bisweilen reaktiv (mitunter fast nur noch disziplinarisch) auf Änderungen der Interessenlagen und Motivationsbrüche bei den Jugendlichen angepasst. Schließlich konzentriert sich bei diesem Typ die Schülerbetreuung auf eine Unterrichtsdurchführung, die auch eine (begrenzte) inhaltliche Begleitung jener Lernsequenzen einschließt, die außerhalb des Berufskollegs etwa als betriebliche Praktika ablaufen. Weitere lebensweltliche Probleme der Jugendlichen bleiben jedoch ausgeblendet. 


\subsubsection{Der Typ einer relativ stark ausgeprägten Institutionalisierung}

Der zweite Idealtyp zeichnet sich dadurch aus, dass die Aufgabe Ausbildungsvorbereitung einen eigenen Organisationsrahmen im Berufskolleg, bestenfalls in einer eigenen Abteilung, erhält. Verbunden ist damit ein kontinuierlicher Einsatz der Lehrkräfte in diesem Bereich und die Reduzierung eines Einsatzes an mehreren Lernorten für wenige Fachlehrer. In Konsequenz dieser institutionellen Verselbstständigung entstehen neue Möglichkeiten der Professionalisierung der Lehrtätigkeit, die auch soweit wie möglich genutzt werden. Vor allem aber entstehen neue Formen der Unterrichtsgestaltung und der Betreuung der Jugendlichen, die eine stärker nachhaltige Bearbeitung ihrer Probleme versprechen.

Bei diesem Typ werden die vorhandenen institutionellen und organisatorischen Spielräume dafür genutzt, die Ziele der Ausbildungsvorbereitung gemäß der Heterogenität von Interessenlagen und Lernvoraussetzungen zu erweitern und neu zu justieren, über externe Kooperation und internes Management diese Heterogenität beherrschbar zu machen und auf der Durchführungsebene ein hohes Maß an Differenzierung, Individualisierung, Flexibilisierung, (berufs-) praktischer Ausrichtung und intensiver Betreuung sicherzustellen.

Es sind vor allem die in der Ausbildungsvorbereitung stark engagierten Berufskollegs, die die im Bereich der nicht Maßnahme gestützten KSoB-Klassen vorhandenen Spielräume für neue Konzeptionen des Bildungsangebots genutzt haben, um in besonderem Maße bestimmten Interessenlagen von Schülerinnen und Schülern wie auch ihren kognitiven und motivationalen Problemen gerecht zu werden. Solche Bildungsangebote, die je nach Zielgruppe in sich weiter differenziert sein können, finden sich vor allem im Bereich der Vollzeit-KSoB-Klassen dieser Kollegs. Hierbei nutzen die Kollegs die Freiräume auch dafür, Zielsetzungen unterhalb der Schwelle eines allgemeinbildenden Abschlusses oder des Übergangs in Ausbildung zu verfolgen; dies gilt vor allem für Jugendliche, für die ein Jahr Ausbildungsvorbereitung zu kurz bemessen erscheint, um Abschluss und Übergang zu gewährleisten.

Diese Form einer stärker an die Ausgangs- und Interessenlage der Jugendlichen anschließenden Erweiterung oder Modifikation des Spektrums an Bildungszielen verbindet sich mit einem ausgeprägten „Heterogenitätsmanagement “ unterrichtsexterner Differenzierung der Schülerschaft: Unterrichtsexterne Differenzierung meint zum einen die von Kollegs mit hohen Schülerzahlen in BOJ- und KSoB-Klassen berichteten „Clearing und Beratungsprozesse“, die zumindest diejenigen Jugendlichen durchlaufen, die sich selbst für ein Berufsorientierungsjahr oder KSoB-Klassen anmelden oder die den Kollegs für die Pflicht-Beschulung im Rahmen der nicht trägergebundenen KsoB-Klassen zugewiesen werden: In persönlichen Beratungsgesprächen mit Jugendlichen (und deren Eltern) wird auf der Grundlage mitgebrachter Zeugnisse, Lebensläufe und Empfehlungen, aber auch aufgrund der geäußerten Interessen der Jugendlichen eine Zuordnung zu einem der Bildungsgänge am Berufskolleg vorgenommen. Dieser liegt in der Regel im Bereich der Ausbildungsvorbereitung, kann aber insbesondere bei Jugendlichen mit besseren Lernvoraussetzungen und ausgeprägten beruflichen Orientierungen auch dazu führen, dass sie in anderen Bildungsgängen (außerhalb der Ausbildungsvorbereitung) aufgenommen werden. Einzelne Kollegs starten diesen Klärungs- und Sortierprozess erst nach Schuljahrsbeginn und setzen ihn später fort; der Prozess bewirkt dann eine gewisse Neusortierung zwischen den einzelnen Angeboten.

Unterrichtsexterne Differenzierung meint darüber hinaus die über die Bildung von Kollegverbünden geschaffenen bzw. erweiterten Möglichkeiten, den Jugendlichen eine Berufsorientierung in den sie interessierenden Berufsfeldern anzubieten - auch wenn das von ihnen zunächst einmal angesteuerte Kolleg hierfür nicht die entsprechende Ausstattung und fachliche Spezialisierung bietet und eine Kooperation mit einem anderen Kolleg gesucht werden muss. Diese Form der Neuzuordnung reduziert jedoch auch dort, wo sie stattfindet, nur begrenzt die Heterogenität in den Klassen der Ausbildungsvorbereitung und damit den offenkundigen Bedarf an intensiver individueller Betreuung und differenziertem Unterricht. Insofern unterbreitet man den Jugendlichen ein individuelles Förder- und Betreuungsangebot.

Auf der Basis dieses Angebots und von kontinuierlichen Förderplangesprächen, die in individuellen „Kontrakten“ münden, werden realistische, individuelle Lernziele erarbeitet, vereinbart und nachgehalten. Die Jugendlichen mit ähnlichen Zielsetzungen werden dann im Rahmen eines Klassenverbandes einer bestimmten Lerngruppe, die jeweils spezifische Aufgaben bearbeitet, zugeordnet. Mit Blick auf die Möglichkeit, dass Interessenlagen sich ändern und Motivationsbrüche auftauchen können, werden die Förderpläne kontinuierlich aktualisiert.

Was diesen Typ weiterhin auszeichnet, ist ein ausdifferenziertes Angebot zu lebensweltlichen und praktischen Themen, auf das die Schüler in Form von Beratungsgesprächen oder aber von medial aufbereiteten Informationen in einer Art Selbststudium zurückgreifen können. Damit erhalten sie eine Form der Unterstützung, die es ihnen ermöglicht, „den Kopf für schulische Lernprozesse freizubekommen“. Schließlich geht es bei diesem Typ auch darum, die praktische Betreuung der Schüler über den Unterricht hinaus auf die private Lebenswelt auszudehnen und schulexterne Faktoren einzubeziehen, die Durchhaltevermögen und Bildungserfolg zu gefährden drohen.

In diesem Typ beschränkt sich die Stärkung berufspraktischer Anteile (mit dem Ziel, Berufsorientierung zu ermöglichen wie auch Lernmotivation zu stärken bzw. zu entfachen) nicht auf betriebliche Praktika, sondern auf die Stärkung praktischer Sequenzen an allen Lernorten, also auch in der Fachpraxis in der Werkstatt oder im Rahmen von Projektunterricht oder Projekttagen bzw. berufs- 
bezogenem Unterricht. Hierbei betonen Schulleitungen und Lehrkörper übereinstimmend die Motivationsschübe und darüber hinaus die Fortschritte in der Entwicklung personaler und sozialer Kompetenzen, die durch gemeinsame Fachpraxis und Projektarbeit ausgelöst werden und offenbar ein gerüttelt $\mathrm{Ma}$ an Anerkennung vermitteln. Solche positiven Entwicklungen werden angestoßen etwa bei der selbstständigen Suche der Praktikumsplätze („,erster Schritt in die Selbstständigkeit“) bis hin zur Eingewöhnung in eine betriebliche und berufliche Sozialorganisation mit ihren unhintergehbaren Anforderungen an übergreifende Tugenden wie „Verlässlichkeit“"wie auch an spezifische Fähigkeiten und Fertigkeiten wie etwa manuelles Geschick.

Vermittlungserfolge scheinen den Typ „stärker ausgeprägte Institutionalisierung" zu bestätigen. In einzelnen Kollegs wurde von einer Vermittlungsquote bis zu $70 \%$ berichtet. Aber auch jenseits von Vermittlungsquoten wurden positive Effekte angeführt. ${ }^{8}$

\subsection{Kompetenz und Professionalisierung des Lehrpersonals}

Die Schilderungen der Arbeit von den Lehrkräften verweisen auf ein Tätigkeitsprofil in der Ausbildungsvorbereitung, das weit über Wissensvermittlung in den Fachklassen dualer Berufsausbildung hinausweist, d.h. komplexer als das der dort eingesetzten Fachlehrer erscheint. Auch wenn die in einer der Diskussionen gefallene, zugespitzte Beschreibung „Mischung von Fachlehrer und Sozialarbeiter" nur einen Aspekt der Komplexitätszunahme - das hohe Maß an individueller Betreuungsarbeit mit Jugendlichen aus schwierigen sozialen Umgebungen - hervorhebt, so verweist sie doch zugleich auf ein Kompetenzprofil, das sich nicht ohne Weiteres nach bestimmten Seiten auflösen bzw. separieren und damit einer arbeitsteiligen Spezialisierung zuführen lässt.

Es ist ein hochkomplexes Kompetenzprofil, in den sich berufsfachliche und allgemeinbildende Kompetenzen (wegen Vermittlung eines allgemeinbildenden Abschlusses) mit Fähigkeiten für eine ansatzweise psychologische Diagnostik von individuellen Verhaltensschwierigkeiten wie auch von der Gruppendynamik in Klassen, Motivationsmanagement in der Unterrichtsgestaltung und sozialpädagogische Kompetenzen für die Einbeziehung von sozialem und familialem Umfeld verbinden. Diese erschöpfen sich nicht in Wissen, sondern müssen durch kommunikative Sensibilität und Erfahrung im Umgang mit „schwierigen“ Jugendlichen aktiviert werden.

Bei der Komplexität des Kompetenzprofils verwundert nicht, dass sich praktisch kein Kolleg findet, in dem nicht ein Mangel an Professionalität und Kompetenzausstattung der Schule für diese Aufgabe artikuliert wurde. Und selbst in den avanciertesten Institutionalisierungsmodellen mit eigener Abteilungs- und Team-Bildung sowie Weiterbildung wurde eine stärker systematische Professionalisierung reklamiert.

\section{Perspektive der Neugestaltung der Ausbildungs- vorbereitung}

Die empirische Analyse der gegenwärtigen Situation der Ausbildungsvorbereitung von Jugendlichen mit besonderem Förderbedarf hat gezeigt, dass eine gesetzliche und untergesetzliche Neugestaltung der Ausbildungsvorbereitung nur dann die gewünschten Erfolge einer besseren Eingliederung der Jugendlichen in Ausbildung erzielen kann, wenn sie begleitet wird von einer stärkeren Institutionalisierung dieser Aufgabe in den Berufskollegs und einer intensiveren Professionalisierung der Lehrkräfte. Ohne hier ein Programm der faktischen Neugestaltung, das zwischen politischen Instanzen und den an der Ausbildungsvorbereitung beteiligten Akteuren auszuhandeln ist, skizzieren zu können, seien einige Richtungshinweise gegeben, die sich aus den empirischen Befunden aufdrängen: Eine unabdingbare Re-Institutionalisierung der Ausbildungsvorbereitung hat die ganze Prozesskette des Übergangs und ihre institutionellen Akteure in den Blick zu nehmen.

Eine bessere Institutionalisierung und Professionalisierung der Berufsvorbereitung erfordert in den Kollegs eine Verankerung dieser Aufgabe in eigenen fachlichen Einheiten, die Kontinuität, Qualität und Professionalität der Aufgabenwahrnehmung sichert. Unabdingbar, aber schwer zu realisieren sind eine Didaktik und Methodik der Ausbildungsvorbereitung, in der allgemeine und berufsorientierende Kompetenzen möglichst integriert vermittelt werden, anstatt sie als zwei im Unterrichtsalltag gegeneinander ausspielbare Bildungsgänge zu verstehen. Ausbildungsvorbereitung erfordert darüber hinaus ein hohes Maß an Differenzierung und Individualisierung der Vermittlungsprozesse sowie eine systematische Institutionen übergreifende Kooperation der Akteure; dies schließt auch die Möglichkeit ein, den Prozess - entsprechend den Ausgangsvoraussetzungen der Jugendlichen - über den Zeitraum eines Jahres hinaus strecken zu können. Das alles zu erreichen, setzt flexible Handlungsspielräume in den Berufskollegs und eine neue Aus- und Weiterbildung der Lehrkräfte voraus.

8 Die berichteten Quoten und Effekte konnten zwar nicht kontrolliert werden, es spricht aber nichts gegen ihre Richtigkeit. Hier ist darauf hinzuweisen, dass in Zukunft Verläufe und Kompetenzzuwächse systematisch erhoben werden sollten, was allerdings mit einem beträchtlichen empirischen Aufwand verbunden ist.

\section{LITERATUR}

Autorengruppe BIBB/Bertelsmann Stiftung (2011): Reform des Übergangs von der Schule in die Berufsausbildung. Aktuelle Vorschläge im Urteil von Berufsbildungsexperten und Jugendlichen, http://www. bertelsmann-stiftung.de/cps/ rde/xbcr/SID-60AA91E6-5D8C0FC2/bst/ Ergebnisbericht\% 20Expertenmonitor\% 202010.pdf

Autorengruppe Bildungsberichterstattung (Hrsg.) (2008): Bildung in Deutschland 2008. Ein indikatorengestützter Bericht mit einer Analyse zu Übergängen im Anschluss an den Sekundarbereich I, Bielefeld

Autorengruppe Bildungsberichterstattung (Hrsg.) (2012): Bildung in Deutschland 2012. Ein indikatorengestützter Bericht mit einer Analyse zur kulturellen Bildung im Lebenslauf, Bielefeld

AVO-RL (2005): Verordnung zur Ausführung des § 93 Abs. 2 Schulgesetz (VO zu § 93 Abs. 2 SchulG) vom 18. März, GV. NRW. S. 218, http://www.schulminis terium.nrw.de/BP/Lehrer/ Lehrerversorgung/FAQ_Lehrerversorgung/AVO.pdf Baethge, M./Baethge-Kinsky, V. (2012): Zur Situation und Perspektiven der Ausbildungsvorbereitung von Jugendlichen mit besonderem Förderbedarf in NRW, Göttingen/Düsseldorf

Beicht, U./Friedrich, M./Ulrich, J. G. (2008) (Hrsg.): Ausbildungschancen und Verbleib von Schulabsolventen, Bielefeld

Blien, U./Hirschenauer, F. (2005): Vergleichstypen 2005, Neufassung der Regionaltypisierung für Vergleiche zwischen Agenturbezirken, IAB-Forschungsbericht (24), Nürnberg

Bundesagentur für Arbeit (BA) (2009): Fachkonzept Berufsvorbereitende Bildungsmaßnahmen vom 20.11., http://www.arbeitsagentur.de/zentraler-Content/HEGA-Internet/A05-Berufl-Qualifizierung/ Publikation/HEGA-11-2009-VAErg-BvB-Fachkonzept-Anlage-2.pdf 
Bundesministerium für Bildung und Forschung (BMBF) (2005): Berufliche Qualifizierung Jugendlicher mit besonderem Förderbedarf, http://www.bmbf. de/pub/berufliche_qualifizierung_jugendlicher.pdf

Eckhardt, Ch./Sommer, J. (2011): Programmbewertung Werkstattjahr, Endbericht, Berlin/Duisburg

Information und Technik Nordrhein-Westfalen (IT NRW) (o.J.): Schulstatistik Krüger-Charlé, M./Leisering, B./Rolff, K. (2011): Vorstudie für eine systematische Aufnahme und Bewertung von Maßnahmen im Übergang von der Schule in Ausbildung und Beruf in Nordrhein-Westfalen, Abschlussbericht, Gelsenkirchen, http://www.iat.eu/aktuell/veroeff/2011/gew.pdf

Kupka, P./Wolters, M. (2010): Erweiterte vertiefte Berufsorientierung, Überblick, Praxiserfahrungen und Evaluationsperspektiven, IAB Forschungsbericht (10), Nürnberg

Lippegaus-Grünau, P./Mahl, F./Stoltz, I. (2011): Berufsorientierung - Programme und Projekte von Bund, Ländern, Kommunen und Stiftungen im Überblick, München

Ministerium für Arbeit und Integration (MAIS) (2011): Merkblatt zur Förderung des Werkstattjahres 2011/2012, Stand 7. Juli 2011, http://www.gib.nrw.de/ service/downloads/WSJ_Merkblatt.pdf

Ministerium für Schule und Weiterbildung (MSW) (2011a): Entwurf APO-BK vom 14.2., Düsseldorf

Ministerium für Schule und Weiterbildung (MSW) (2011b): Ausbildungs- und Prüfungsordnung Berufskolleg (APO-BK) Stand: 01.07.2011, http://www.schulministerium.nrw.de/BP/Schulrecht/APOen/APOBK.pdf

Organisation für wirtschaftliche Entwicklung und Zusammenarbeit (OECD) (Hrsg.) (2010): Was Schülerinnen und Schüler wissen und können: Schülerleistungen und Lesekompetenz, Mathematik und Naturwissenschaften, Bd. 1, Bie lefeld

Plicht, H. (2010): Das neue Fachkonzept berufsvorbereitender Bildungsmaßnahmen der BA in der Praxis, IAB Forschungsbericht (07), http://doku.iab.de/ forschungsbericht/2010/fb0710.pdf

Solga, H./Kohlrausch, B./Fromm, S. (2010): Evaluation des Projekts

Abschlussquote erhöhen - Berufsfähigkeit steigern", IAB Forschungsbericht

(5), Nürnberg, http://doku.iab.de/forschungsbericht/2010/fb0510.pdf
Statistisches Bundesamt (2011): Schnellmeldung integrierte Ausbildungsberichterstattung 2010, https://www.destatis.de/DE/Publikationen/Thematisch/BildungForschungKultur/Schulen/ SchnellmeldungAusbildungsberichterstattung5211002107004.pdf?_blob=publicationFile

Statistisches Bundesamt (2012): Schnellmeldung integrierte Ausbildungsberichterstattung 2011, https://www.destatis.de/DE/Publikationen/Thematisch/BildungForschungKultur/Schulen/ SchnellmeldungAusbildungsberichterstattung . html

Zentrum für Europäische Wirtschaftsforschung (ZEW)/RambolI/INFAS (2010): Vorstudie zur Evaluation von Fördermaßnahmen für Jugendliche im SGB II und SGB III, Bundesministerium für Arbeit und Soziales, Forschungsbericht 405, Berlin

\section{AUTOREN}

MARTIN BAETHGE, Prof. Dr., ist Präsident des Soziologischen Forschungsinstituts (SOFI) an der Universität Göttingen. Arbeitsschwerpunkte: Bildungssoziologie mit den Schwerpunkten berufliche Aus- und Weiterbildung, Arbeitssoziologie (Dienstleistungsforschung).

martin.baethge@sofi.uni-goettingen.de

VOLKER BAETHGE-KINSKY, Dr., ist wissenschaftlicher Mitarbeiter am SOFI. Arbeitsschwerpunkte: Berufsbildungsforschung, Arbeitsmarktforschung.

volker.baethge@sofi.uni-goettingen.de 